\title{
Reperfusion after Acute Coronary Occlusion in Dogs Impairs Endothelium-dependent Relaxation to Acetylcholine and Augments Contractile Reactivity In Vitro
}

\author{
Karyl M. VanBenthuysen, Ivan F. McMurtry, and Lawrence D. Horwitz \\ Division of Cardiology and Cardiovascular-Pulmonary Research Laboratory, University of Colorado \\ Health Sciences Center, Denver, Colorado 80262
}

\begin{abstract}
Endothelial injury may contribute to the augmented coronary vascular tone seen in myocardial ischemia by impairing endothelial production or release of vasodilators. In vitro reactivity of arterial rings was studied after $60 \mathrm{~min}$ of coronary occlusion and $60 \mathrm{~min}$ of reperfusion in anesthetized dogs. Ischemia without reperfusion blunted contractile reactivity to potassium chloride (KCl), whereas ischemia plus reperfusion augmented contractile responses to both $\mathrm{KCl}$ and ergonovine. The response to acetylcholine, an endothelium-dependent vasodilator, was abolished in reperfused arteries, whereas the response to nitroprusside, an endothelium-independent vasodilator, was intact. Verapamil pretreatment restored $\mathrm{KCl}$ contractile responses to normal in reperfused coronary rings and partially restored endotheliumdependent relaxation. Electron microscopy revealed a nondenuding epicardial coronary endothelial injury in reperfused arteries. These data support the hypothesis that reperfusion of ischemic myocardium augments reactivity to vasoconstrictor agents by causing endothelial cell damage, excessive calcium influx, and loss of modulating vasodilator function.
\end{abstract}

\section{Introduction}

Coronary artery "spasm" has been identified as the basis of "variant" or "Prinzmetal" angina $(1,2)$, as a precipitant of myocardial infarction (3), and as a frequent factor in unstable and postinfarction angina (4-6). The mechanism by which this abnormal form of coronary vasoconstriction occurs is not known. However, recent interest has focused on the vascular endothelium as a determinant of vasodilator tone. Furchgott described a chemical "relaxing" factor, which is produced by endothelial cells and vasodilates coronary and other systemic arteries (7). Damage to the endothelium may impair release of this relaxing factor or other endothelial-derived vasodilators, and cause vasoconstriction. Recent studies have assessed the role of the endothelium in modulating coronary vascular tone by mechanically removing the endothelium of large, epicardial coronary arteries. Deendothelialization produces spontaneous local vasoconstric-

Address reprint requests to Dr. VanBenthuysen, Division of Cardiology, 111B Veterans Administration Medical Center, 1055 Clermont St., Denver, CO 80220.

Presented at the Western Society for Clinical Investigation, February 1985 and the National Meeting of the American Federation for Clinical Research, May 1985, and published in abstract form (1985. Clin. Res. 33:91a;523a.). 1986.

Received for publication 14 January 1986 and in revised form 7 July

J. Clin. Invest.

(c) The American Society for Clinical Investigation, Inc.

$0021-9738 / 87 / 01 / 0265 / 10 \quad \$ 1.00$

Volume 79, January 1987, 265-274 tion (8), enhances reactivity to vasoconstrictors such as 5-hydroxytryptamine $(8,9)$, and impairs endothelial-dependent relaxation to acetylcholine (7), 5-hydroxytryptamine (9) and thrombin (10). Thus, although gross disruption of the coronary endothelium may have important effects on vascular tone, it is unclear whether less severe forms of endothelial injury can similarly influence vasoreactivity.

Changes in vasoreactivity similar to those produced by removal of endothelium were recently described by $\mathrm{Ku}(10)$ in a canine model of myocardial infarction and reperfusion. Rings removed from an occluded and reperfused coronary artery exhibited enhanced reactivity and impaired relaxation when exposed in vitro to thrombin. We speculated that such changes were not specific for thrombin but resulted from a reperfusion related endothelial injury. To test this hypothesis, we studied coronary reactivity in vitro using arteries from a canine model of myocardial infarction and reperfusion. We observed that occlusion followed by reperfusion, but not occlusion alone, augmented reactivity of coronary rings from the affected region. Reperfusion was associated with ultrastructural evidence of endothelial injury, and there was an impaired endothelial-dependent relaxation. Coronary occlusion and reperfusion, a common pathologic event, thus may enhance coronary vascular reactivity by causing endothelial injury.

\section{Methods}

Canine model of myocardial ischemia and reperfusion. 32 mongrel dogs of either sex, weighing $15-24 \mathrm{~kg}$, were sedated with intramuscular morphine sulfate after which anesthesia was induced with thiopental and maintained with chloralose. An endotracheal tube was inserted and the dogs were mechanically ventilated. Arterial blood gases were measured, and supplemental inspired oxygen, ventilatory rate, and tidal volume were adjusted to maintain $\mathrm{pH}$ at 7.35-7.45, $\mathrm{PO}_{2}>75 \mathrm{mmHg}$ and $\mathrm{PCO}_{2}$ 34-40 mmHg. The electrocardiogram was continuously monitored. A left thoracotomy was performed through the fifth intercostal space, and the heart was suspended in a pericardial cradle. The left anterior descending coronary artery was identified, and was carefully dissected free immediately distal to the origin of the first large diagonal branch. In most cases, this dissection site occurred between 2 and $4 \mathrm{~cm}$ from the origin of the anterior descending artery. The artery was snared with surgical silk, the free ends passed through a flexible rubber tube, and this "rubber shod" secured with a surgical clamp during occlusion. Coronary occlusion rapidly produced a cyanotic appearance in the region subtended by the occluded coronary artery and was associated with dyskinetic anterior wall motion and typical electrocardiographic manifestations of ischemia. Reperfusion was accomplished by release of the coronary artery ligature. Ventricular arrhythmias occurring after occlusion or reperfusion were not treated. Ventricular fibrillation occurred in only 1 of 32 dogs, and that animal was excluded from analysis.

Isolated coronary vascular rings. After either coronary occlusion or occlusion plus reperfusion, the beating hearts were rapidly excised and placed in a physiologic salt solution (composition given below) at $4^{\circ} \mathrm{C}$. The left anterior descending artery was isolated by sharp dissection and a section at least $2 \mathrm{~cm}$ distal to the ligature was removed. A section of 
the proximal circumflex was also isolated and removed. Using microdissection instruments, the periadventitial tissue was removed, while avoiding manipulation of the arterial intima. One to four arterial rings were cut using paired surgical blades fixed $2 \mathrm{~mm}$ apart. The rings were placed in a muscle bath containing physiologic salt solution. The bath was aerated at $37^{\circ} \mathrm{C}$ with a gas mixture of $21 \%$ oxygen, $5 \% \mathrm{CO}_{2}$, and $74 \%$ nitrogen, which produced a pH of 7.44, $\mathrm{PO}_{2}$ of 128 torr and $\mathrm{PCO}_{2}$ of 24 torr. The intact rings were mounted on stainless steel hooks suspended from force displacement transducers (Statham Instruments, Inc., Oxnard, CA) that permitted measurement of tension parallel to the circular smooth muscle cell elements. Resting tension was adjusted to $4 \mathrm{~g}$. In separate experiments, optimal tension of $\mathbf{4} \mathbf{g}$ was defined as that tension at which contractile responses to $5 \mathrm{~min}$ exposure to $50 \mathrm{mM} \mathrm{KCl}$ were maximal. There were no differences in optimal tension between anterior descending and circumflex arteries, nor were there differences between ischemic/reperfused and control arteries. Input from the transducers was displayed on a multichannel recorder (Grass Instrument Co., Quincy, MA). A typical time delay from excision of the heart to placement of the arterial rings in the bath was $10 \mathrm{~min}$. The coronary rings were allowed to equilibrate for 60 min before administration of vasoactive drugs. In some experiments, the endothelium was removed by gently abrading the arterial intima with a tapered wooden stick. Deendothelialization was confirmed functionally by loss of the endothelium-dependent relaxation response to acetylcholine.

Experimental protocol. The dogs were divided into two groups. Group I (ischemia alone) consisted of dogs that underwent $60 \mathrm{~min}$ of left anterior descending occlusion, after which the heart was excised and the coronary artery rings were collected. Group II (ischemia + reperfusion) consisted of dogs that underwent coronary occlusion for $60 \mathrm{~min}$ followed by 60 min of reperfusion before excision of the heart. $60 \mathrm{~min}$ was selected because previous investigators (11) have shown that briefer periods of ischemia were not associated with elevated coronary vascular resistance. Furthermore, in six preliminary experiments, we have measured regional coronary vascular resistance after $60 \mathrm{~min}$ of ischemia followed by reperfusion. Although the initial response was coronary vasodilation, vascular resistance began to rise by $30 \mathrm{~min}$ of reperfusion. Thus, in an identical model, coronary vascular resistance was increased after $60 \mathrm{~min}$ of ischemia and $60 \mathrm{~min}$ of reperfusion. For each heart one or two rings were cut from the left anterior descending artery at least $2 \mathrm{~cm}$ distal to the ligature and these served as experimental rings. One or two rings were cut from the circumflex artery and these served as control rings. When duplicate rings were studied in our four-bath apparatus, the responses were averaged and included in the final data analysis. The occasional problem of damage to the vascular rings during artery harvesting was avoided by not including rings with contractile responses of $<1.5 \mathrm{~g}$ to an initial bolus of $40 \mathrm{mM} \mathrm{KCl}$. Less than $10 \%$ of rings were excluded by this criteria, and there were no differences in "nonreactive" rings between groups. In preliminary experiments $(n=4)$, contractile responses to potassium chloride $(\mathrm{KCl})$ and relaxation responses to acetylcholine were the same in the left anterior descending and same in the left anterior descending and circumflex artery rings from dogs not subjected to coronary ligation.

Contractile responses were measured using cumulative dose responses to $\mathrm{KCl}$ ( $n=$ number of dogs; control, $n=14$; ischemia alone, $n=9$; ischemia + reperfusion, $n=7$ ) and ergonovine maleate (control, $n=12$; ischemia alone, $n=7$; ischemia + reperfusion, $n=5$ ). Vasodilator responses after precontraction with $30 \mathrm{mM} \mathrm{KCl}$ were measured using both the endothelial-dependent vasodilator, acetylcholine (control, $n=22$; ischemia alone, $n=11$; ischemia + reperfusion, $n=10$; deendothelialization, $n=13)$, and the direct smooth muscle vasodilator, sodium nitroprusside (control, $n=4$; ischemia + reperfusion, $n=4$; deendothelialization, $n=4)$.

To assess the role of excessive calcium influx in mediating reperfusion related changes in vascular reactivity, we treated five dogs during coronary artery occlusion and reperfusion with verapamil $(0.1 \mathrm{mg} / \mathrm{kg}$ bolus, followed by a $0.01 \mathrm{mg} / \mathrm{kg}$ per min intravenous infusion). Potassium chloride dose responses (control, $n=5$; ischemia + reperfusion, $n=5$ ) and ace- tylcholine responses (control, $n=5$; ischemia + reperfusion, $n=5$ ) were then measured. Verapamil was not added to the tissue baths.

To assess the influence of vasodilator prostaglandins on the blunted vascular reactivity observed in the ischemic coronary rings, the cyclooxygenase blocker, meclofenamate $(1 \mu \mathrm{g} / \mathrm{ml})$, was added to the tissue bath. This concentration has been shown to be effective in inhibiting prostaglandin biosynthesis in comparable in vitro systems.

Electron microscopy. In six dogs, the coronary vasculature was fixed for electron microscopy following completion of ischemia with or without reperfusion. The beating heart was rapidly excised and the ascending aorta cannulated. The aortic root was retrogradely perfused at a controlled pressure of 100 torr, with $1.5 \%$ glutaraldehyde in $0.1 \mathrm{M}$ cacodylate buffer, $\mathrm{pH}$ 7.3. After $10 \mathrm{~min}$ of perfusion, a single $2-3-\mathrm{cm}$ segment was removed from both the left anterior descending and the circumflex arteries. Two arteries from each of six dogs thus were analyzed. These segments were submerged in cacodylate buffered glutaraldehyde for $24 \mathrm{~h}$ at $4^{\circ} \mathrm{C}$. The arteries were subdivided into $1-2 \mathrm{~mm}$ pieces and post-fixed in $1 \%$ osmium tetroxide in $0.1 \mathrm{M}$ cacodylate buffer, $\mathrm{pH} 7.3$ at $4^{\circ} \mathrm{C}$ for $1 \mathrm{~h}$. En bloc $1 \%$ aqueous uranyl acetate staining was performed and then tissues were dehydrated in acetone, cleared in propylene oxide, and infiltrated with Embed/araldite overnight. The embedded arteries were cured $2 \mathrm{~d}$ at $70^{\circ} \mathrm{C}$. Sections were cut on an Ultrotome (LKB Instruments, Inc., Gaithersburg, $\mathrm{MD}$ ), and stained with 3\% aqueous uranyl acetate and Reynold's lead stain. Up to four thick sections from the Embed-araldite-infiltrated blocks were examined, following which one to two thin sections were examined on an electron microscope (400T, Philips Medical Systems, Inc., Shelton, CT) at an accelerating voltage of $60 \mathrm{kV}$.

Preparation of reagents and stock solutions. $\mathrm{KCl}$ (Sigma Chemical Co., St. Louis, MO) was prepared in an $18.6 \%$ solution and administered in aliquots resulting in final bath concentrations of 5-80 mM. Ergonovine maleate (Sigma Chemical Co.) was administered in doses sufficient to produce final bath concentrations of $10^{-8}$ to $5 \times 10^{-6} \mathrm{M}$. Acetylcholine (Sigma Chemical Co.) was prepared as a stock solution of $2 \times 10^{-3} \mathrm{M}$ and administered to achieve final concentrations from $10^{-7}$ to $10^{-6} \mathrm{M}$. Verapamil hydrochloride (Searle, Chicago, IL) was diluted with $0.9 \%$ saline and administered as a $0.1-\mathrm{mg} / \mathrm{kg}$ bolus followed by a $0.01 \mathrm{mg} / \mathrm{kg}$ per min infusion. Sodium nitroprusside (Elkins-Sinn, Inc., Cherry Hill, $\mathrm{NJ}$ ) was prepared in a $2 \times 10^{-4} \mathrm{M}$ solution and administered so as to achieve final bath concentrations of $10^{-8}$ to $5 \times 10^{-7} \mathrm{M}$. Reagents used to prepare the physiologic salt solution were obtained from Sigma Chemical Co. and the final concentrations were as follows: $\mathrm{NaCl}(119$ $\mathrm{mM}), \mathrm{KCl}(4.7 \mathrm{mM}), \mathrm{MgSO}_{4}: 7 \mathrm{H}_{2} \mathrm{O}$ (1.17 mM), $\mathrm{NaHCO}_{3}$ (22.6 mM), $\mathrm{KH}_{2} \mathrm{PO}_{4}(1.18 \mathrm{mM}), \mathrm{CaCl}_{2}: 2 \mathrm{H}_{2} \mathrm{O}(3.2 \mathrm{mM})$, sucrose $(50 \mathrm{mM})$, and glucose (5.5 mM). Meclofenamate (Warner-Lambert Co., New York) was dissolved in distilled water and stored refrigerated as a stock solution of $0.1 \%$. The ergonovine, acetylcholine, and sodium nitroprusside solutions were prepared fresh daily.

Statistical analysis. Dose-response curves to $\mathrm{KCl}$ and ergonovine were plotted for each vascular ring and normalized for maximal tension developed. Graphic determination of $\mathrm{ED}_{\mathbf{5 0}}$ (the concentration of drug required to elicit $50 \%$ of maximal contraction) was obtained from dose response curves and the $\mathrm{ED}_{\mathbf{s 0}}$ was used as a measure of "reactivity." Vasodilator responses were quantitated using a "relaxation index," defined as the ratio of drug induced relaxation to the precontracted tension. Comparisons among groups were made using a one-way analysis of variance with the Student-Newman-Keul multiple comparison test.

\section{Results}

Vascular reactivity to potassium chloride. Contractile responses to cumulative administration of $\mathrm{KCl}$ are shown in Fig. $1 . \mathrm{KCl}$ caused a curvilinear increase in tension developed by the isolated coronary artery rings; the mean $\mathrm{ED}_{50}$ for the control rings was $29.4 \pm 2.8 \mathrm{mM}$. Rings obtained from coronary arteries subjected to ischemia without reperfusion were less reactive with a rightward shift of the dose response curves; the $\mathrm{ED}_{50}$ was $37.7 \pm 3.3$ 


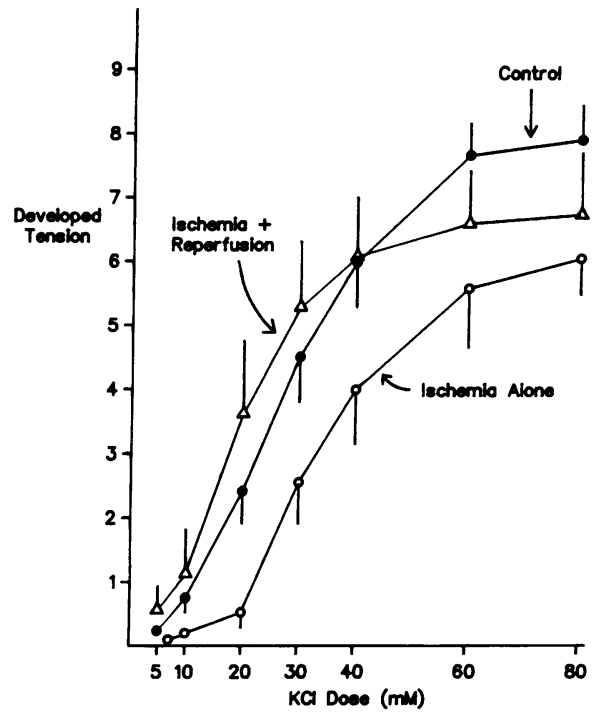

Figure 1. Contractile responses of isolated coronary artery rings to $\mathrm{KCl}$. Tension (in grams) in control rings increased in a curvilinear manner $\left(\mathrm{ED}_{50}=29.4 \mathrm{mM}, n=14\right)$. This curve was right shifted in rings from coronary arteries subjected to occlusion (ischemia alone; $\mathrm{ED}_{50}=37.7 \mathrm{mM}, n=9$ ). Reperfusion (ischemia + reperfusion) caused a significant left-shifting of the dose-response curve $\left(\mathrm{ED}_{50}\right.$ $=20.7 \mathrm{mM}, n=7$ ), implying enhanced reactivity to $\mathrm{KCl}$.

$\mathrm{mM}$, which was significantly different from control curves. In contrast, in rings obtained from coronary arteries subjected to $60 \mathrm{~min}$ of reperfusion following the ischemia, the curves were shifted to the left compared to control and the $\mathrm{ED}_{50}$ was $20.7 \pm 2.5$ $\mathrm{mM},(P<0.05)$, indicating augmented reactivity to $\mathrm{KCl}$. Meclofenamate $(1 \mu \mathrm{g} / \mathrm{ml})$ restored the $\mathrm{KCl}$ contractile response in the ischemic coronary artery rings to the control level (Fig. 2).

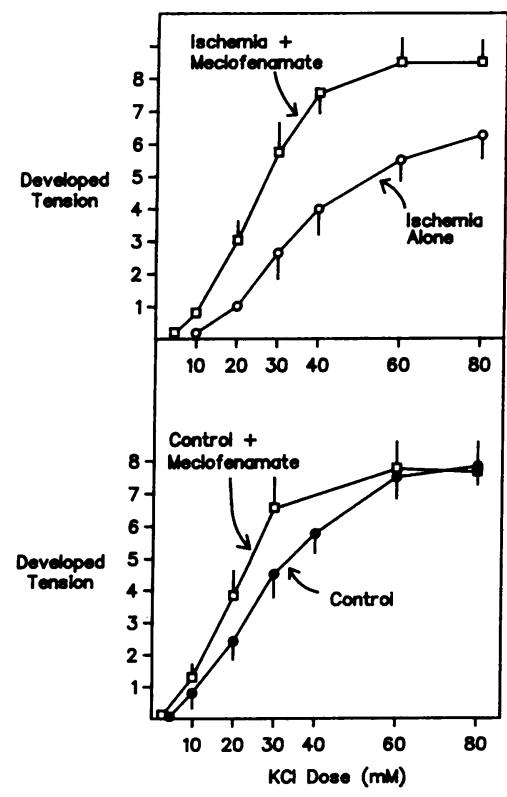

Figure 2. Effect of meclofenamate on contractile responses of control and ischemic coronary artery rings. The top panel illustrates the effect of meclofenamate on contractile responses of rings from ischemic coronary arteries, and the bottom panel illustrates the effect of meclofenamate on control rings. Developed tension (g). Meclofenamate caused a marked leftshifting $(P<0.05)$ of the $\mathrm{KCl}$ dose-response curve in ischemic rings $\left(\mathrm{ED}_{50}=24.8 \mathrm{mM}, n\right.$ $=5$ ) compared to ischemic rings without meclofenamate $\left(\mathrm{ED}_{\mathbf{5 0}}\right.$

$=37.7 \mathrm{mM}, n=9)$. In contrast, the effect of meclofenamate on control rings was less (control $\mathrm{ED}_{50}=29.4 \mathrm{mM}, n=14 \mathrm{vs}$. control $/ \mathrm{me}-$ clofenamate $\mathrm{ED}_{50}=21.9 \mathrm{mM}, n=8$ ).

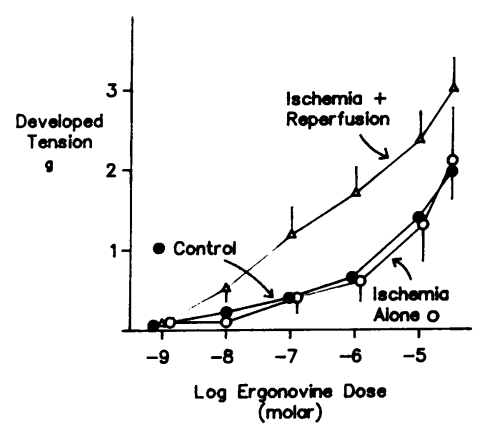

Figure 3. Contractile responses of isolated coronary artery rings to ergonovine. The curvilinear dose-response curve of control arterial rings $\left(\mathrm{ED}_{\mathbf{5 0}}=3.4\right.$ $\times 10^{-6} \mathrm{M}, n=12$ ) was unchanged in rings from ischemic coronary arteries $\left(\mathrm{ED}_{50}=5.4 \times 10^{-6} \mathrm{M}, n\right.$ $=7$ ), whereas reperfusion caused a significant leftshifting of the dose response curve to ergonovine $\left(\mathrm{ED}_{50}\right.$ $=1.13 \times 10^{-6} \mathrm{M}, n=5$ ).
Meclofenamate also caused a small but significant increase in vascular reactivity in the control coronary vascular rings (Fig. 2), although the magnitude of this change was less than that seen in ischemic rings and the $\mathrm{ED}_{50}$ values for meclofenamate control and meclofenamate ischemic arteries did not differ statistically. Thus, with respect to contractile responses to $\mathrm{KCl}$ there was increased reactivity of vessels exposed to ischemia and reperfusion and decreased reactivity of vessels exposed to ischemia alone; the blunted vascular reactivity in ischemic vessels may have been due to prostaglandin effects.

Vascular reactivity to ergonovine maleate. Dose-response curves to cumulative administration of ergonovine maleate are shown in Fig. 3. There were no significant differences in contractile responses between the control and the ischemic, nonreperfused rings. However, reactivity was increased in the coronary artery rings exposed to ischemia and reperfusion (control $\mathrm{ED}_{50}=3.43 \pm .9 \times 10^{-6} \mathrm{M}$, ischemia $\mathrm{ED}_{50}=5.4 \pm 2.1 \times 10^{-6} \mathrm{M}$, ischemia + reperfusion $\mathrm{ED}_{50}=1.13 \pm .65 \times 10^{-6} \mathrm{M},(P<0.05$ vs. control and ischemia). Therefore, as was the case with $\mathrm{KCl}$, the ischemic and reperfused rings showed increased reactivity to ergonovine. The effects of meclofenamate were not studied during ergonovine administration.

Acetylcholine-induced vascular relaxation. Fig. 4 illustrates the vascular response to acetylcholine. In control coronary artery rings with intact endothelium there was a dose-dependent fall in muscle tension following precontraction with $30 \mathrm{mM} \mathrm{KCl}$. The dependence of this phenomenon on intact endothelial cells was demonstrated when control coronary artery rings that had been denuded of endothelium by mechanical rubbing of the luminal surface did not relax in response to acetylcholine. In coronary artery rings exposed to ischemia without reperfusion there was mild blunting of the relaxation with acetylcholine. The relaxation response to acetylcholine was severely blunted in vessels exposed to both ischemia and reperfusion. In contrast, when rings from vessels subjected to ischemia and reperfusion were challenged with sodium nitroprusside, there was a fall in tension similar to results in control vessels. Cumulative results are shown in Fig. 5. In summary, acetylcholine, which causes vascular relaxation through endothelial-dependent mechanisms, had no effect on reperfused coronary rings, whereas, nitroprusside, an endothelial-independent vasodilator, caused normal relaxation in these coronary rings.

Effect of deendothelialization on vascular reactivity. Contractile dose response curves to $\mathrm{KCl}$ are shown in Fig. 6. In coronary artery rings that had been mechanically deendothe- 


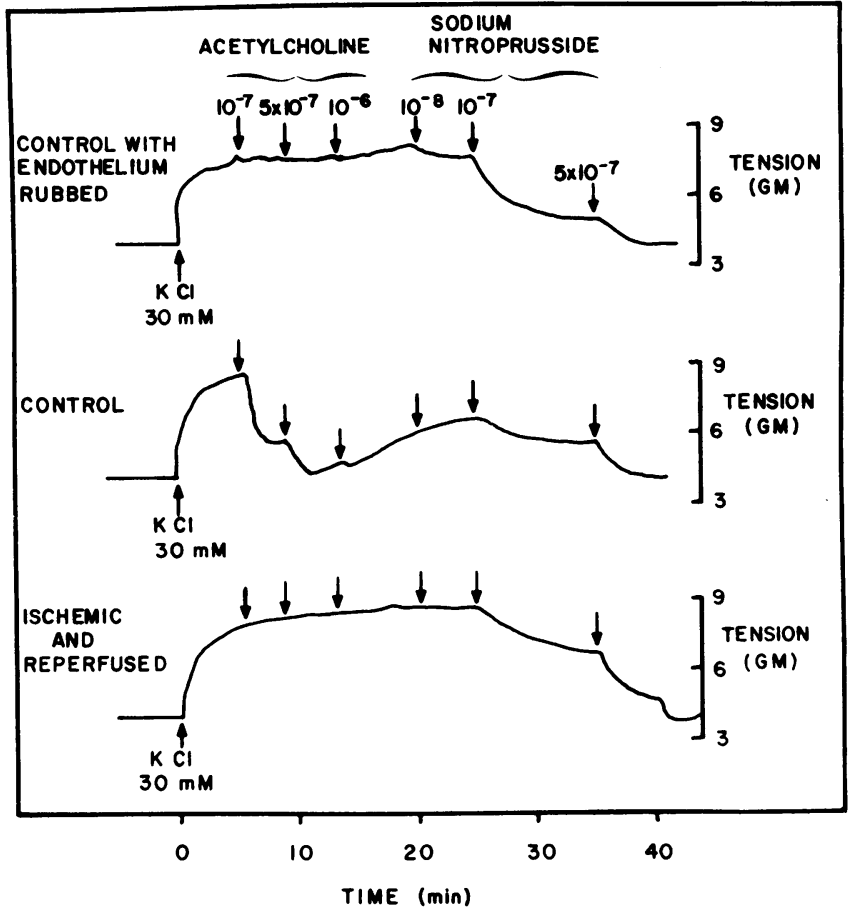

Figure 4. Endothelial-dependent relaxation of isolated coronary artery rings. Raw data curves from a single experiment are shown. Acetylcholine caused a prompt relaxation of control coronary rings with intact endothelium following precontraction with $30 \mathrm{mM} \mathrm{KCl}$. Mechanical deendothelialization or exposure to ischemia and reperfusion abolished this endothelial-dependent relaxation. However, all coronary artery rings relaxed normally in response to sodium nitroprusside, a direct vascular smooth muscle dilator. Tension measured in grams.

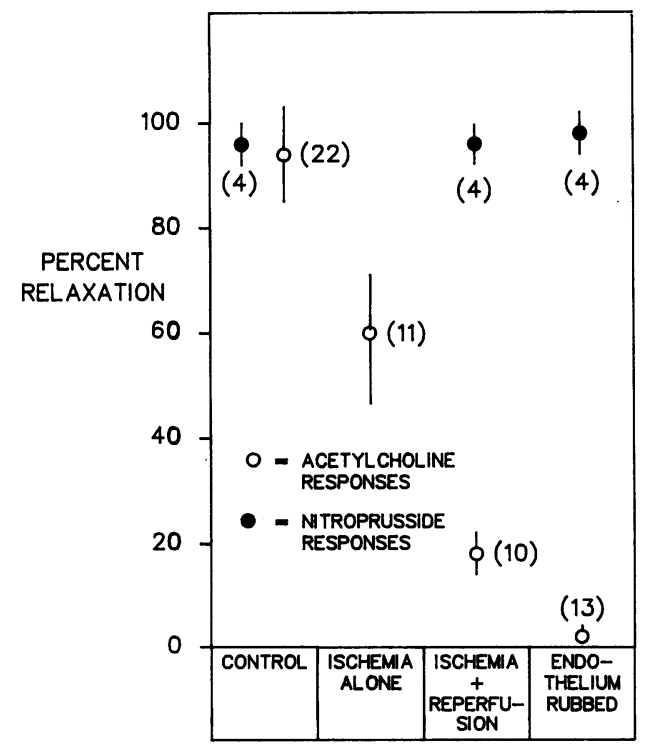

Figure 5. Acetylcholine and nitroprusside relaxation in isolated coronary artery rings. Acetylcholine completely relaxed intact arterial rings precontracted with $\mathrm{KCl}$. Although this relaxation was mildly blunted in rings exposed to ischemia, it was virtually abolished in rings exposed to ischemia and reperfusion. Mechanical de-endothelialization mimicked the changes seen in ischemic and reperfused rings. In contrast, nitroprusside completely relaxed all rings tested.

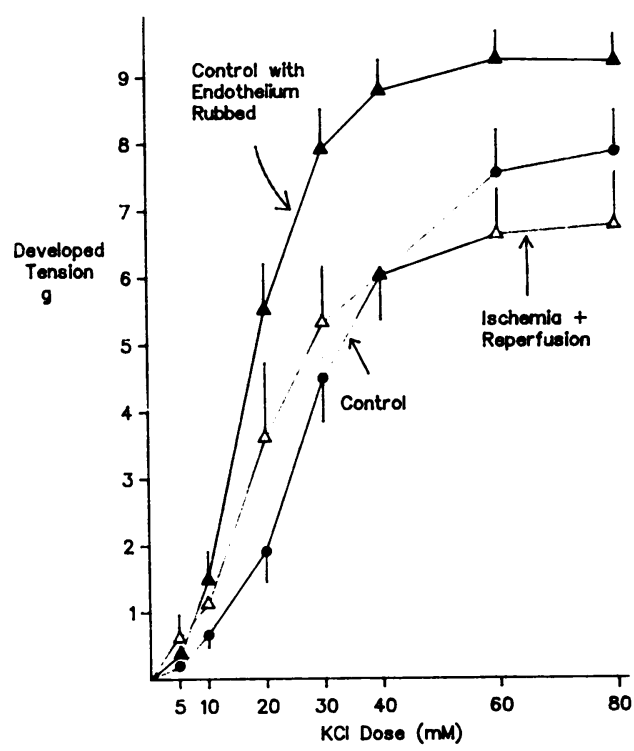

Figure 6. Effect of mechanical deendothelialization on contractile responses to $\mathrm{KCl}$ in coronary artery rings. The dose response curves in control $\left(\mathrm{ED}_{50}=29.4 \mathrm{mM}, n=14\right)$ and ischemia/reperfused rings $\left(E_{50}=20.7 \mathrm{mM}, n=7\right)$ are again shown for reference. Mechanical deendothelialization further shifted the curve to the left $\left(\mathrm{ED}_{50}=18.8\right.$ $\mathrm{mM}, n=9$ ) implicating enhanced reactivity to $\mathrm{KCl}$.

lialized, there was a left shifting of the contractile response to $\mathrm{KCl}\left(\mathrm{ED}_{50}=18.8 \pm 2.0 \mathrm{mM}, P<0.05\right)$, compared to both control and reperfused coronary artery rings. Thus, mechanical deendothelialization mimicked the effect of ischemia and reperfusion on $\mathrm{KCl}$ responses.

Effect of verapamil pretreatment on reperfusion-induced change in vascular reactivity. Five dogs were treated with verapamil before coronary artery occlusion and throughout the period of ischemia and reperfusion; verapamil was not added to the isolated tissue bath. Fig. 7 compares the contractile responses

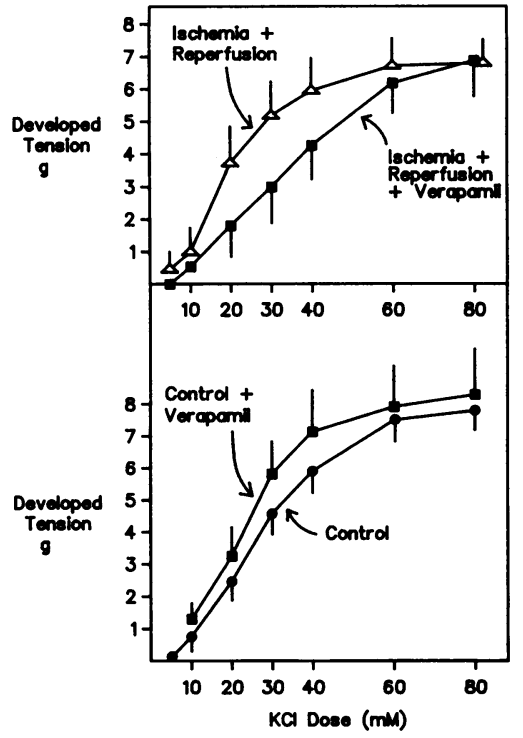

Figure 7. Effect of verapamil pretreatment on coronary artery ring reactivity. In vivo treatment of control arteries with verapamil had no effect on the $\mathrm{KCl}$ reactivity (control $\mathrm{ED}_{50}$ $=29.4 \mathrm{mM}, n=14 \mathrm{vs}$. control + verapamil $\mathrm{ED}_{50}=26.0 \mathrm{mM}, n$ $=5, P=\mathrm{NS}$ ) (bottom panel). However, verapamil pretreatment significantly right shifted the $\mathrm{KCl}$ dose-response curves of arteries exposed to ischemia and reperfusion (ischemia/ reperfusion $\mathrm{ED}_{50}=29.7$ $\mathrm{mM}, n=7$ vs. ischemia/reperfusion + verapamil $\mathrm{ED}_{50}=32.0$ $\mathrm{mM}, n=5 ; P<0.05)$. 


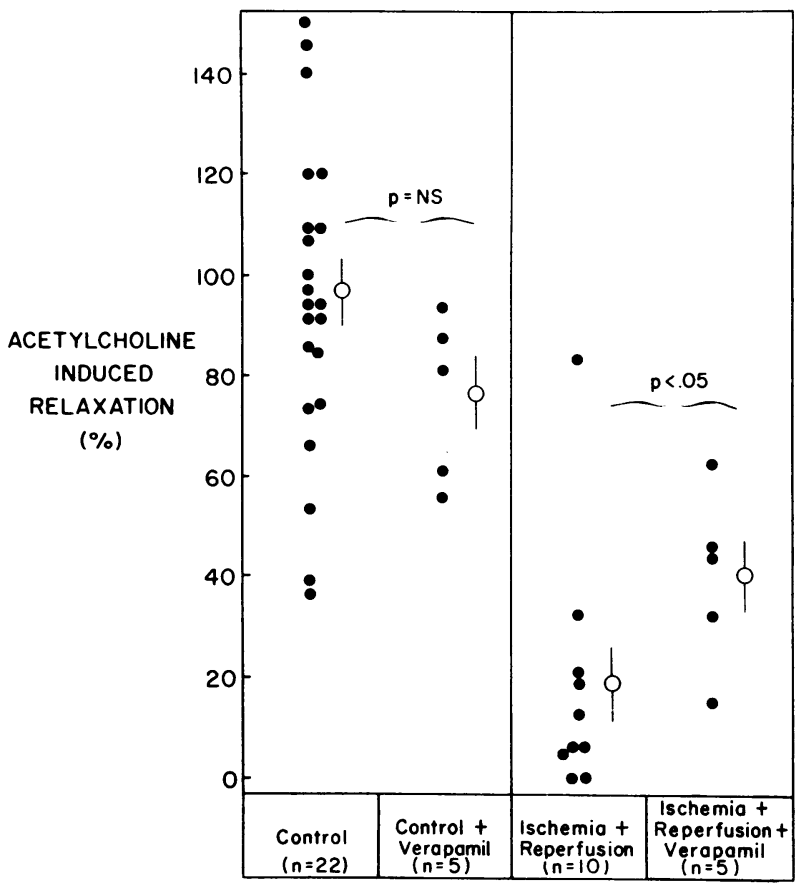

Figure 8. Effect of verapamil pretreatment on endothelial-dependent relaxation. In vivo treatment with verapamil partially restored the endothelial-dependent response to acetylcholine in ischemic and reperfused coronary artery rings, but had no effect on control rings. to $\mathrm{KCl}$ in control and reperfused vessels collected from dogs with and without verapamil pretreatment. Whereas verapamil pretreatment caused a rightward shift, to normal levels, of the dose-response curve of reperfused artery rings, it had no effect on vascular reactivity to $\mathrm{KCl}$ in control rings. The effect of verapamil pretreatment on acetylcholine-induced relaxation is shown in Fig. 8. Although verapamil mildly blunted relaxation to acetylcholine in control rings, the acetylcholine-induced relaxations following ischemia and reperfusion were partially restored with verapamil pretreatment. Therefore, pretreatment with a calcium entry blocker entirely prevented the increase in vascular reactivity and limited the impairment in relaxation of arteries exposed to ischemia and reperfusion.

Electron microscopic findings. Coronary arteries from six dogs were prepared for electron microscopic evaluation of endothelial cell injury (Figs. 9-13). Endothelial cells from control vessels contained prominent nuclei, were closely adherent to the underlying subendothelium, and had intact intercellular junctions. Samples from ischemic coronary arteries contained scattered subendothelial blebs and poor definition of intracellular organelles, but no gross disruption of intercellular junctions or evidence of separation of the endothelial cells from the underlying subendothelium. Rare, focal areas of endothelial cell degeneration were observed (Fig. 10). The electron micrographs taken from coronary arteries exposed to ischemia and reperfusion showed evidence of early endothelial cell loss including subendothelial bleb formation, disrupted intercellular junctions, and
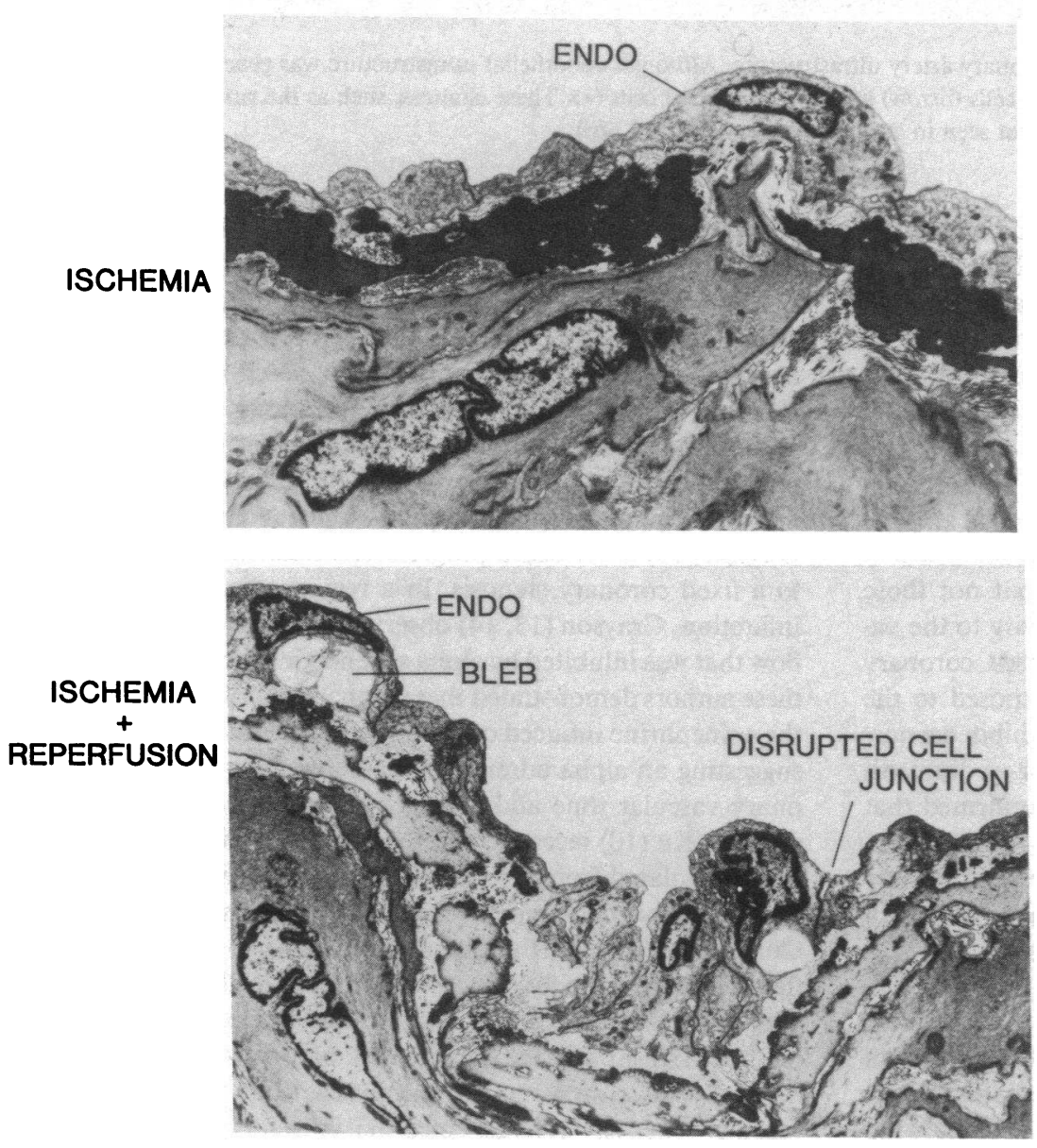

Figure 9. Electron microscopic changes in ischemic and ischemic/reperfused coronary arteries. Coronary arteries exposed to ischemia without reperfusion demonstrated an intact endothelial cell layer $(E N D O)$ closely adherent to the underlying internal elastic lamina. The nuclear pattern and intercellular junctions were intact. Coronary arteries exposed to ischemia and reperfusion showed focal areas of heaped up endothelium, subendothelial bleb formation $(B L E B)$ and disruption of intercellular junctions. 


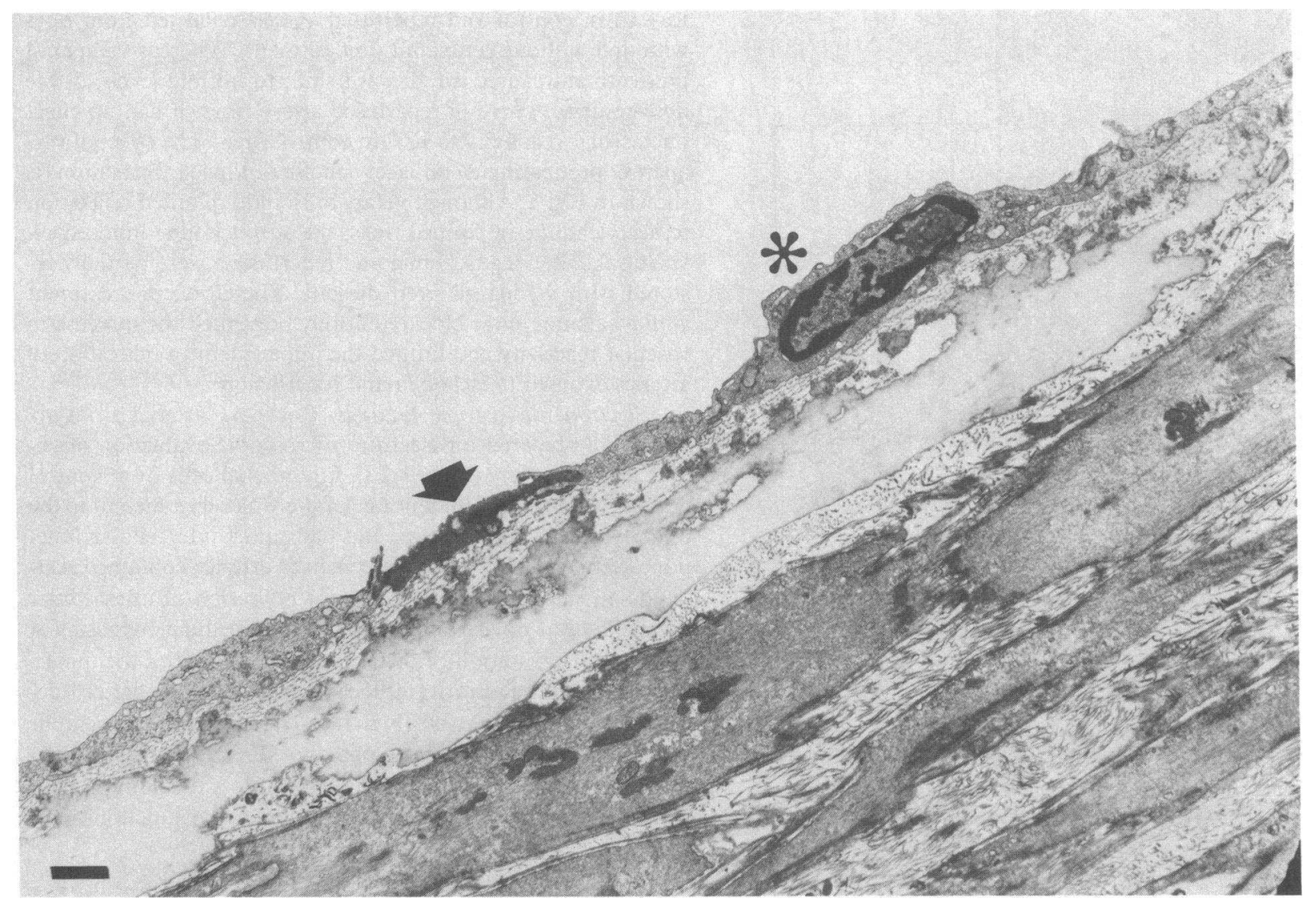

Figure 10. Effect of ischemia without reperfusion on coronary artery ultrastructure. Although endothelial ultrastructure was generally normal, there were isolated examples of degenerating endothelial cells (arrow) adjacent to normal cells (*). These changes, such as the ruffled, indistinct cell membrane and the poorly defined cell organelles, were not seen in control arteries. (Bar $=1 \mu \mathrm{m})$.

heaped up endothelium, changes not seen in ischemic or control arteries (Fig. 12). Platelet and polymorphonuclear leukocyte adherence to endothelium of reperfused arteries was also observed (Fig. 13). Minor ultrastructural changes thus were noted with ischemia alone, but when both ischemia and reperfusion occurred, there was major damage to the vascular endothelium.

\section{Discussion}

Vascular rings isolated from segments of dog coronary artery subjected in vivo to ischemia and reperfusion, but not those subjected to ischemia alone, had increased reactivity to the vasoconstrictors $\mathrm{KCl}$ and ergonovine. The reperfused coronary artery rings also did not relax normally when exposed to the endothelial-dependent dilator acetylcholine but exhibited a normal response to the direct smooth muscle dilator, sodium nitroprusside. Transmission electron microscopy confirmed that reperfusion caused a nondenuding injury to the endothelium. Reperfusion of transiently ischemic coronary arteries thus may enhance coronary vascular reactivity, and perhaps coronary vascular resistance, because of endothelial damage.

Although there is increased coronary vascular resistance following release of a coronary artery obstruction, it is not clear whether this is due to augmented coronary vascular tone (10, 12-15) or to microvascular obstruction by intravascular thrombosis or leukostasis (16), endothelial cell swelling (11) or extrinsic compression from myocardial edema $(11,17)$. Recent studies have supported a role for augmented coronary vascular tone during reperfusion. In a model of partial coronary artery occlusion, Gorman and Sparks (12) observed progressive increases in resistance that were not explained by vasoconstrictor prostaglandins, potassium ion or norepinephrine. Crystal (15) reported coronary pressure-volume data during reperfusion and interpreted these results as inconsistent with microvascular obstruction but compatible with abnormal vascular control mechanisms. Guyton (18) described a progressive impairment in flow distal to a fixed coronary stenosis. In a region adjacent to an acute infarction, Grayson $(13,14)$ observed a progressive fall in blood flow that was inhibited by alpha adrenergic blockade. Moreover, these authors demonstrated that coronary artery ligation reversed the epinephrine induced coronary vasodilation seen preligation, suggesting an alpha adrenergic mediated augmentation in coronary vascular tone and loss of normal beta adrenergic vasodilation. $\mathrm{Ku}(10)$ recently reported that large epicardial coronary arteries isolated from a region subjected to ischemia and reperfusion exhibited increased contractile responses to thrombin. Our study extended the observation of $\mathrm{Ku}$ by demonstrating that the altered reactivity was not unique to thrombin and by suggesting that reperfusion-induced endothelial injury may be the mechanism for the augmented coronary vasoconstrictor reactivity. It is important to emphasize that these changes in vascular reactivity of large, epicardial coronary arteries may not 


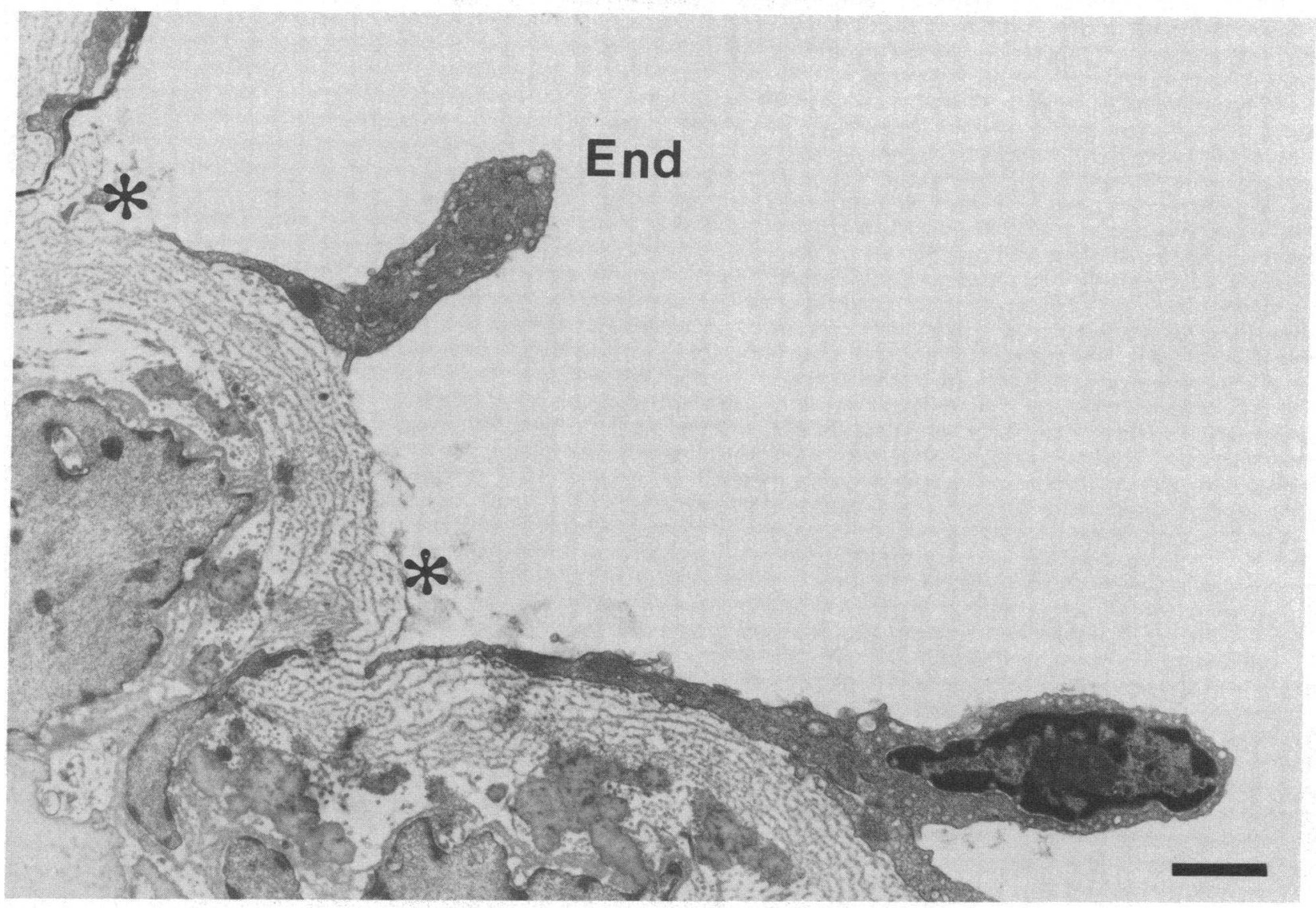

Figure 11. Effect of ischemia plus reperfusion on coronary endothelial ultrastructure. Reperfusion-related changes included focal areas of endothelial cell injury with partial detachment from the underlying subendothelium (asterisk). (Bar $=1 \mu \mathrm{m})$.

reflect similar processes in the arteriolar, or resistance vessels of the reperfused myocardium. However, it is likely that a reperfusion injury may account for the experimental observations of augmented coronary vascular resistance cited above $(12-15,19)$. Even isolated epicardial endothelial injury and hyperreactivity may assume clinical importance, however, as small changes in epicardial artery tone superimposed on major lumenal stenoses may cause significant reductions in coronary blood flow.

There is considerable evidence that injury to the coronary endothelium may augment coronary vascular reactivity. Balloon catheter inflation has been associated with augmented epicardial coronary vasoconstriction to 5-hydroxytryptamine in anesthetized dogs (8). Using a model of chronic endothelial injury produced by balloon catheter inflation and an atherogenic diet in pigs, Shimokawa observed augmented coronary vasoconstrictor responses to histamine, 5-hydroxytryptamine and ergonovine (20). The same authors also reported enhanced canine, epicardial vasoconstriction to ergonovine which persisted 6 mo after balloon catheter injury and an atherogenic diet (21).

A plausible explanation for the association of augmented vascular reactivity and endothelial injury is loss of modulating vasodilator tone. The description by Furchgott of a "relaxing factor" produced by the endothelium following the administration of agents such as acetylcholine (7) provided considerable insight into the role of the endothelium in modulating vascular smooth muscle function. To determine if coronary endothelial injury impaired acetylcholine induced endothelium-dependent relaxation, Schipke (22) infused intracoronary acetylcholine to anesthetized dogs before and after removal of endothelium produced by the local infusion of distilled water. Acetylcholine caused both epicardial and resistance vessel vasodilation under control conditions, but epicardial coronary vasoconstriction after endothelial injury. Our study indicated that coronary artery occlusion and reperfusion inhibited acetylcholine-induced, endothelium-dependent relaxation, and this impaired vasodilation was not associated with gross deendothelialization. This observation is consistent with impaired production of endothelialdependent relaxing factor, however a defect in the endothelial cell receptor for acetylcholine cannot be excluded.

An alternative explanation for loss of modulating vasodilator function following endothelial injury is loss of vasodilator prostaglandin production. The arterial endothelium is a rich source of prostacyclin $\left(\mathrm{PGI}_{2}\right)(23)$ a potent vasodilator and antiplatelet agent. The importance of prostacyclin in modulating coronary vascular tone was recently demonstrated by Lane and Bove (19). Balloon catheter injury of proximal coronary arteries produced no change in outer diameter or total flow. However, under conditions of cyclooxygenase inhibition, endothelial injury caused a time-dependent epicardial vasoconstriction up to $50 \%$ of the cross sectional diameter. Although the importance of the loss of vasodilator prostaglandin production was not specifically addressed in our study, $\mathrm{Ku}(10)$ found that the augmented reactivity 


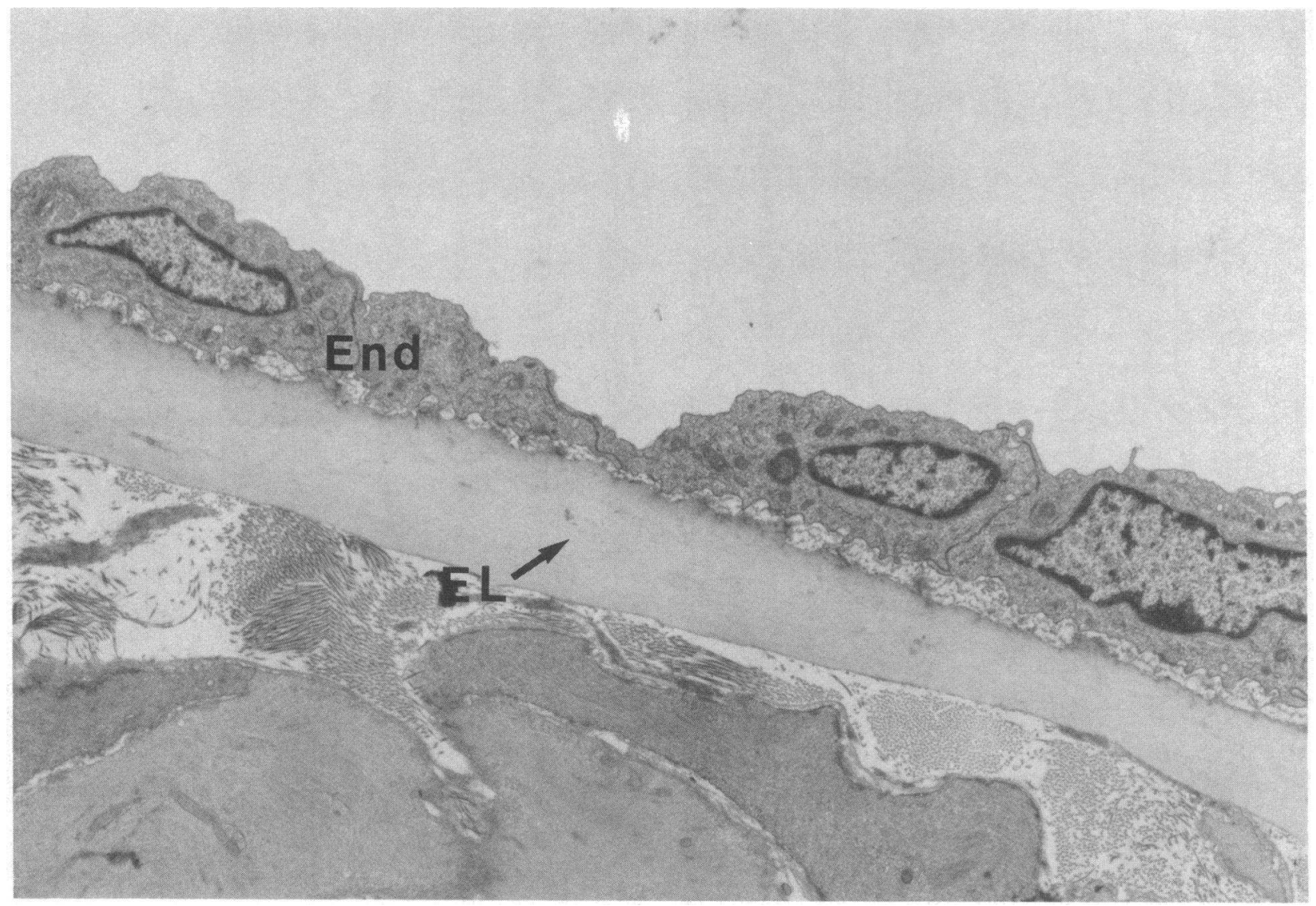

Figure 12. Normal coronary endothelial ultrastructure. Control artery endothelial cells $(E N D)$ were flat in profile with intact cytoplasmic membranes, distinct intracellular organelles, and tight intercellular junctions. There were no areas of endothelial cells dislodged from the underlying subendothelium or internal elastic lamina $(E L)$.

of ischemic and reperfused coronary rings was not affected by the cyclooxygenase blocker, indomethacin.

The mechanism of reperfusion-mediated endothelial injury is not clear. Potential mechanisms include platelet- or leukocyteendothelial interactions (Fig. 13), increased shear stress superimposed on ischemic endothelial injury, and oxygen free radicals derived from neutrophils or generated upon reoxygenation of ischemic tissue. After the initial endothelial membrane injury, it is likely that cytosolic calcium accumulation exacerbates the endothelial damage. Reperfusion of myocardium irreversibly injured by ischemia causes striking increases in myocyte calcium (24), an event correlated with membrane damage (25). Moreover, the use of membrane-protective agents or calcium influx inhibitors in experimental models of myocardial ischemia and reperfusion has reduced infarction size and limited ventricular dysfunction (26-28). Therefore, we reasoned that inhibition of excessive calcium influx could modify the coronary vascular injury in our model of ischemia plus reperfusion. We found that treatment with the calcium entry blocker, verapamil, during ischemia and reperfusion blocked the augmented reactivity to $\mathrm{KCl}$ and attenuated the impaired endothelial-dependent relaxation. Verapamil restored $\mathrm{KCl}$ reactivity and acetylcholine-induced relaxation to the pre-reperfusion levels (Fig. 14) further supporting the role of reperfusion-mediated coronary endothelial injury.

In contrast to the augmented vascular reactivity of isolated coronary artery rings following ischemia and reperfusion is the blunted reactivity seen following ischemia alone. Meclofenamate restored vascular reactivity to normal, implicating a potential role for ischemia-related vasodilator prostaglandin production $(29,30)$. Although meclofenamate also shifted the control dose response curve leftward, the magnitude of this increase in reactivity was considerably less than that in ischemic rings.

Our findings may be relevant to certain clinical phenomena involving the process known as "coronary artery spasm." Myocardial ischemia due to coronary artery spasm in the early postinfarction period has been described (4-6). Hyperreactivity to ergonovine is common in patients with recent myocardial infarctions but rare in patients tested months or years after a myocardial infarction (31). Although these observations were made in patients who were not exposed to physician-initiated interventions leading to rapid reperfusion, endogenous thrombolytic mechanisms can cause spontaneous reperfusion (32). The current widespread clinical use of thrombolytic agents such as streptokinase, urokinase, or tissue plasminogen activator in the early stages of acute myocardial infarction may lead to an increased incidence of reperfusion injury to the coronary artery endothelium. If so, there may be a high occurrence of postinfarction coronary artery spasm in patients who have received such treatment. The favorable response to a calcium blocker in our canine model encourages the possibility that pharmacologic interventions that diminish endothelial cell damage could prevent or attenuate myocardial perfusion abnormalities following relief of acute coronary artery obstructions. 


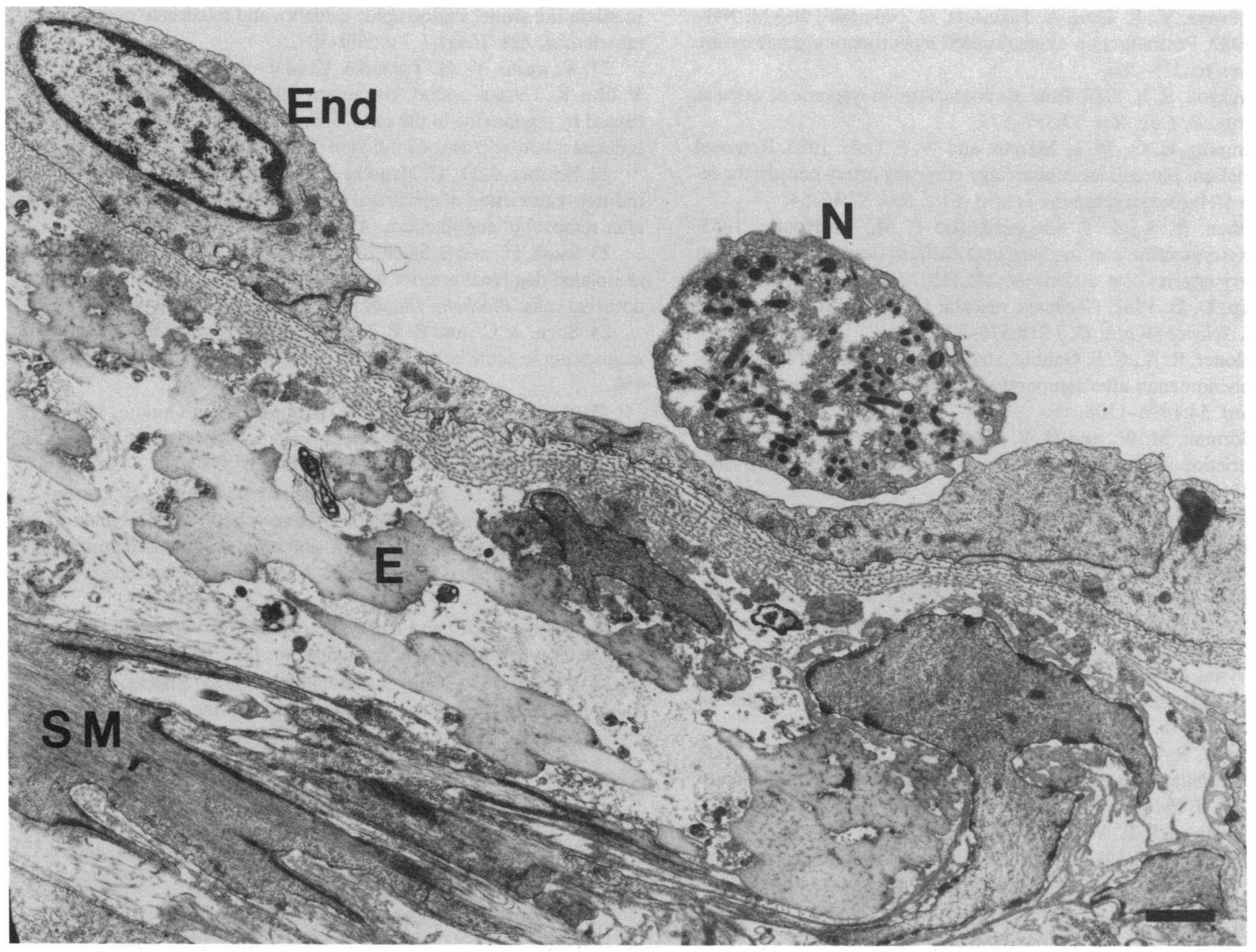

Figure 13. Effect of ischemia plus reperfusion on coronary endothelial ultrastructure. Adherence of circulating blood elements such as neutrophils $(N)$ to the endothelium of ischemic and reperfused arteries was seen.

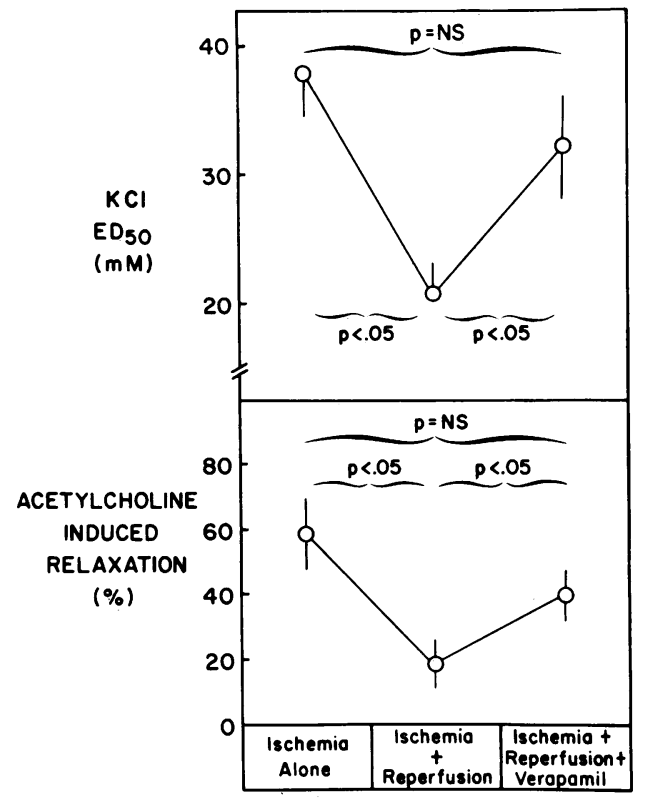

Figure 14. Effect of verapamil on reactivity to $\mathrm{KCl}$ and acetylcholineinduced relaxation. Verapamil pretreatment restored vascular reactivity toward the ischemic level, and partially restored the endothelial-dependent relaxation.

\section{Acknowledgment}

The authors gratefully acknowledge the expert technical assistance of Mr. Stephen Bell, Ms. Holly Coller, and the patient secretarial skills of Ms. Karen Mileham. We wish to thank Dr. Jan Hensen of the National Jewish Center for Respiratory Medicine and Immunology for her advice and support with the electron microscopy. The artwork was skillfully prepared by Mr. Stephen Hoffmeister, Ms. Rosann McCullough, and Ms. Eva Toyos.

This work was supported by the generous donations of a group of businessmen in Denver, CO.

\section{References}

1. Hillis, L. D., and E. Braunwald. 1978. Coronary artery spasm. $N$. Engl. J. Med. 299:695-702.

2. Maseri, A., S. Chierchia, and A. L'Abbate. 1980. Pathogenetic mechanisms underlying the clinical events associated with atherosclerotic heart disease. Circulation. 62(Suppl. 5):3-13.

3. Oliva, P. B., and J. C. Breckinridge. 1977. Arteriographic evidence of coronary artery spasm in acute myocardial infarction. Circulation. $56: 366-374$.

4. Nakamura, M., and Y. Koiwaya. 1983. Effect of diltiazem on recurrent spontaneous angina following acute myocardial infarction. Circ. Res. 52(Suppl. 1):I158-I162.

5. Stenson, R. E., M. D. Flamm, Jr., B. L. Zaret, and R. L. MoGowan. 1975. Transient ST-segment elevation with postmyocardial infarction angina: prognostic significance. Am. Heart J. 89:449-454. 
6. Koiwaya, Y., S. Torii, A. Takeshita, O. Nakagaki, and M. Nakamura. 1982. Postinfarction angina caused by coronary arterial spasm. Circulation. 65:275-280.

7. Furchgott, R. F. 1983. Role of endothelium in response of vascular smooth muscle. Circ. Res. 53:557-573.

8. Lamping, K. G., M. L. Marcus, and W. P. Dole. 1985. Removal of endothelium potentiates canine large coronary artery constrictor response to 5-Hydroxytryptamine in vivo. Circ. Res. 57:46-54.

9. Cohen, R. A., J. T. Shepherd, and P. M. VanHoutte. 1983. 5-Hydroxytryptamine can mediate endothelium-dependent relaxation of coronary arteries. Am. J. Physiol. 245:H1077-H1080.

10. Ku, D. D. 1982. Coronary vascular reactivity after myocardial ischemia. Science (Wash. DC) 218:576-578.

11. Kloner, R. A., C. E. Ganote, and R. B. Jennings. 1974. The "noreflow" phenomenon after temporary coronary occlusion in the dog. $J$. Clin. Invest. 54:1496-1508.

12. Gorman, M. W., and H. V. Sparks. 1982. Progressive coronary vasoconstriction during relative ischemia in canine myocardium. Circ Res. 51:411-420.

13. Grayson, J. 1966. Observations on the mechanisms of infarction in the dog after experimental occlusion of the coronary artery. Lancet. 1:1284-1288.

14. Grayson, J., M. Irvine, J. R. Parratt, and J. Cunningham. 1968. Vasospastic elements in myocardial infarction following coronary occlusion in the dog. Cardiovasc. Res. 2:54-62.

15. Crystal, G. J., H. F. Downey, and F. A. Bashour. 1982. Pressureinduced changes in coronary flow and volume during reperfusion in canine hearts. Clin. Exp. Pharmacol. Physiol. 9:485-494.

16. Engler, R. L., G. W. Schmid-Schoenbein, and R. Pavela. 1983. Leukocyte capillary plugging in myocardial ischemia and reperfusion in the dog. Am. J. Pathol. 111:98-111.

17. Willerson, J. T., W. P. Powell, T. E. Guiney, J. J. Stark, C. A. Sanders, and A. Leaf. 1972. Improvement in myocardial function and coronary blood flow in ischemic myocardium after mannitol. J. Clin. Invest. 51:2989-2998.

18. Guyton, R. A., J. H. McClenatnan, and L. L. Michaelis. 1977. Evolution of regional ischemia distal to a proximal coronary stenosis: self-propagation of ischemia. Am. J. Cardiol. 40:381-392.

19. Lane, G. E., and A. A. Bove. 1985. The effect of cyclooxygenase inhibition on vasomotion of proximal coronary arteries with endothelial damage. Circulation. 72:389-396.

20. Shimokawa, H., H. Timoike, S. Nabeyama, H. Yamamoto, Y. Ishii, K. Tanaka and M. Nakamura. 1985. Coronary artery spasm induced in miniature swine: angiographic evidence and relation to coronary atherosclerosis. Am. Heart J. 110:300-309.

21. Kawachi, Y., H. Tomoike, Y. Marvoka, Y. Kikuchi, H. Araki, Y. Ishii, K. Tanaka, and M. Nakamura. 1984. Selective hypercontraction caused by ergonovine in the canine coronary artery under conditions of induced atherosclerosis. Circulation. 69:441-450.

22. Schipke, J. D., G. Heusch, and V. Thamar. 1985. Acetylcholine induces constriction of epicardial coronary arteries in anesthetized dogs after removal of endothelium. Arzneim.-Forsch. 35:926-929.

23. Satoh, $H$., and $S$. Satoh. 1984. Prostaglandin $E_{2}$ and $I_{2}$ production in isolated dog renal arteries in the absence or presence of vascular endothelial cells. Biochem. Biophys. Res. Comm. 118:873-876.

24. Shen, A. C., and R. B. Jennings. 1972. Myocardial calcium and magnesium in acute ischemic myocardial injury. Am. J. Pathol. 67:417440.

25. Whalen, D. A., Jr., D. G. Hamilton, C. E. Ganote, and R. B. Jennings. 1974. Effect of a transient period of ischemia on myocardial cells. I. Effects on cell volume regulation. Am. J. Pathol. 74:381-398.

26. Tilton, R. G., E. K. Williamson, P. A. Cole, K. B. Larson, C. Kilo, and J. R. Williamson. 1985. Coronary vascular hemodynamic and permeability changes during reperfusion after no-flow ischemia in isolated, diltiazem-treated rabbit hearts. J. Cardiovasc. Pharmacol. 7:424436.

27. Reimer, K. A., and R. B. Jennings. 1984. Verapamil in two reperfusion models of myocardial infarction. Lab. Invest. 51:655-666.

28. Reimer, K. A., and R. B. Jennings. 1985. Effects of calciumchannel blockers on myocardial preservation during experimental acute myocardial infarction. Am. J. Cardiol. 55:107b-115b.

29. Berger, H. J., B. Zaret, L. Speroff, L. S. Cohen, and S. Wolfson. 1976. Regional cardiac prostaglandin release during myocardial ischemia in anesthetized dogs. Circ. Res. 38:566-571.

30. Needleman, P., S. D. Bronson, A. Wycke, M. Swakoff, and K. C. Nicolaou. 1978. Cardiac and renal prostaglandin $\mathrm{I}_{2}$. J. Clin. Invest. 61: 839-849.

31. Bertrand, M. E., J. M. LaBlanche, P. Y. Tilmant, F. A. Thieuleux, M. R. Delforge, A. G. Carre, P. Asseman, B. Berzin, C. Libersa, and J. Laurent. 1982. Frequency of provoked coronary arterial spasm in 1089 consecutive patients undergoing coronary arteriography. Circulation. 65: 1299-1306.

32. DeWood, M. A., J. Spores, and E. R. Notsk. 1980. Prevalence of total coronary occlusion during the early hours of transmural myocardial infarction. N. Engl. J. Med. 303:908-912. 